\title{
Práctica contemporánea de la angioplastia coronaria en Chile. Informe final del Registro Nacional de Angioplastia Coronaria (RENAC) 2001-2002
}

\author{
G astón Dussaillant $\mathrm{N}^{1,2}$, Eugenio Marchant $\mathrm{D}^{1,3}$, Hernán Donoso $\mathrm{P}^{1,4}$, \\ Mauricio Aninat $\mathrm{H}^{1,5}$, Marcos 0 pazo $\mathrm{L}^{1,6}$, René Pumarino $0^{1,7}$, \\ Juan Delgado D ${ }^{1,8}$, Ronald Kauffmann $Q^{1,9}$, Jorge Escobar $E^{1,10}$, \\ Miguel A C umsille $\mathbf{G}^{11 a}$, Valeria Bordes $\mathrm{T}^{\mathrm{b}}$.

\section{Final report of the Chilean National Coronary Angioplasty Registry (RENAC)}

Background: Coronary angioplasty was introduced in Chile in 1982, but only after the introduction of stents it became commonplace. However, the results of this procedure at the national level remain largely unknown. Aim: To assess the results and characteristics of coronary angioplasty and develop a national registry in Chile (RENAC). Patients and Methods: All centers performing angioplasty were invited to contribute prospectively with the clinical, angiographic, procedural characteristics and results of all consecutive coronary angioplasty procedures attempted between June 2001 and October 2002. Results: In 10 centers, 1768 lesions were treated in 1484 procedures (22.98\% in women). Mean age was $60.8 \pm 11.3$ years old. Diabetes was found in $21.8 \%$, hypertension in $60.2 \%$, dyslipidemia in $52.0 \%$, active smoking in $40.0 \%$, renal failure in $6.2 \%$. Myocardial infarction was recent in $28.4 \%$ and acute in $19.7 \%$. Forty eight percent of patients had unstable angina and $15.5 \%$ had stable angina. Fifty three percent of patients had single vessel disease and 71\%, normal left ventricular eyection fraction. One vessel was treated in $90.6 \%$ of patients and $81.7 \%$ of lesions were treated with stents, $17.9 \%$ only with baloon and in $0.4 \%$ with atherectomy. Angiographic success was obtained in $95.2 \%$. Clinical success was obtained in $92.2 \%$, and $95.1 \%$ in patients without acute myocardial infarction. Overall inhospital death was $2.2 \%$. In patients without myocardial infarction, the figure was $1.1 \%$. Conclusions: Coronary angioplasty in Chile is performed mostly for the treatment of acute coronary syndromes. Stents are the most frequently used devices. The high success, low mortality and complications observed are comparable to North American registries (Rev Méd Chile 2004; 132: 913-22).

(Key Words: Coronary disease; Angioplasty, transluminal, percutaneous Coronary; Stents)

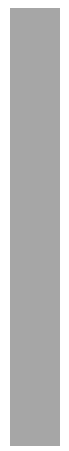

Recibido el 16 de enero, 2004. Aceptado en versión corregida el 6 de julio, 2004.

Trabajo con apoyo de las siguientes empresas: Asicom Medical Systems, representantes de Medtronic; Boston Scientific Chile Ltda; Cardiosol Ltda, representantes de Guidant; Laboratorio Eli Lilly Interamerica Inc; Johnson \& Johnson de Chile SA. (CORDIS); Laboratorio Merck Sharp \& Dohme; Laboratorio Sanofi Synthelabo de Chile SA.

Ninguna de las empresas patrocinadoras arriba mencionadas tuvieron influencia en el diseño del estudio, análisis o recolección de los datos ni revisión o aprobación del manuscrito.

${ }^{1}$ Departamento de Hemodinamia de la Sociedad Chilena de Cardiología y Cirugía Cardiovascular. Laboratorios de Hemodinamia del: ${ }^{2}$ Centro Cardiovascular del Hospital Clínico de la Universidad de Chile, Santiago. ${ }^{3}$ Departamento de Enfermedades Cardiovasculares del Hospital Clínico de la Universidad Católica, Santiago. ${ }^{4}$ Hospital San Juan de Dios, Santiago. ${ }^{5}$ Hospital Gustavo Fricke, Viña del Mar. ${ }^{6}$ Hospital Naval, Viña del Mar. ${ }^{7}$ Hospital Militar, Santiago. ${ }^{8}$ Hospital Clínico de la Fuerza Aérea de Chile, Santiago. ${ }^{9}$ Clínica Las Condes, Santiago y ${ }^{10}$ Hospital Regional de Temuco, Temuco. ${ }^{11}$ Escuela de Salud Pública de la Universidad de Chile, Santiago.

aEstadístico, benfermera Universitaria. 
L a angioplastia coronaria (AC), un procedimiento percutáneo de revascularización, alternativo a la cirugía de puentes coronarios, luego de 26 años de desarrollo, ha pasado a ser la intervención médica más frecuentemente practicada en el mundo. En Chile, la introducción del procedimiento se remonta a $1982^{1}$. Posteriormente hubo un período de abandono, para luego reiniciarse la experiencia hacia fines de la década 1980-89. Sin embargo, sólo la introducción de las prótesis endovasculares o stents, a mediados de la década siguiente, catalizó definitivamente el desarrollo y expansión de estas intervenciones en el país.

La información clínica y científica presentada y publicada a nivel mundial es muy abundante y creciente en el tiempo. En Chile hemos ido a la zaga de lo que ha ido ocurriendo en este aspecto en los países desarrollados. Existen, a nivel nacional, algunas comunicaciones sobre los resultados de los procedimientos de AC, que informan sus características en relación a cuadros clínicos ${ }^{2-6} 0$ técnicas específicas ${ }^{7,8}$ por lo general en centros aislados. No existían esfuerzos a nivel nacional por conocer las características y resultados del procedimiento en el país. Por ello, a fines del año 2000, surge la iniciativa de realizar un gran registro que incorpore la experiencia de todos los centros nacionales en los cuales se practican estas intervenciones, y de esa manera conocer la realidad nacional sobre las características clínicas de los pacientes sometidos a AC y los resultados obtenidos en los procedimientos efectuados.

Los objetivos del presente artículo son dar a conocer el diseño de un registro nacional de AC, las características clínicas, los resultados angiográficos y la evolución intrahospitalaria de todos los pacientes incorporados a este registro, en particular de aquellos que fueron sometidos a AC fuera del contexto del infarto agudo del miocardio. Este último subgrupo de pacientes, por sus características especiales será presentado en forma separada en otro artículo.

\section{Pacientes y Métodos}

Patrocinados por el Departamento de Hemodinamia de la Sociedad Chilena de Cardiología y Cirugía Cardiovascular, en 2001 fueron invitados a partici- par en el Registro Nacional de Angioplastia Coronaria (RENAC), la totalidad de los 20 centros de hemodinamia de adultos chilenos. Uno en Antofagasta, dos en Valparaíso/Viña del Mar, trece en Santiago, uno en Rancagua, dos en Concepción y uno en Temuco. Se les solicitó incorporar al registro a todos los pacientes consecutivos en quienes se intentó efectuar una $\mathrm{AC}$, durante un período ininterrumpido de al menos un año.

Se obtuvo una participación completa con inclusión de todos los casos consecutivos, en sólo 10 centros. De los 10 centros restantes, dos centros no enviaron casos al registro y los restantes enviaron una cantidad menor a los efectuados en el centro correspondiente durante el período de inclusión. De los 10 centros con participación completa, en 4 se comenzó el período de inclusión en marcha blanca, en junio de 2001 y en los 6 restantes se comenzó la inclusión en noviembre del mismo año. El período de inclusión para todos los centros se completó en octubre de 2002. Para efectos del análisis sólo se consideraron los pacientes ingresados en estos 10 centros. En el Apéndice 1 se detallan los centros participantes, investigadores y coordinadores.

En cada centro, la ficha clínica fue llenada en cada procedimiento de AC, ya sea en forma personal por el investigador o bajo su supervisión por un(a) enfermero(a) o tecnólogo(a) entrenado(a). Una enfermera coordinadora a nivel nacional revisó cada ficha en cuanto al llenado completo y consistencia de los datos. Posteriormente, la ficha fue enviada para su ingreso en una base de datos especializada. Finalmente, y previo al análisis de los datos, se procedió a evaluar la falta de datos y consistencia de toda la información incorporada.

Se incorporaron las siguientes variables clínicas: edad, sexo, presencia de diabetes tratada con dieta o medicamentos; hipertensión arterial; dislipidemia (si el paciente tomaba hipolipemiantes o tenía colesterol total $\geq 240 \mathrm{mg} / \mathrm{dL}$ o colesterol de LDL $\geq 160 \mathrm{mg} / \mathrm{dL}$ o colesterol HDL $\leq 25 \mathrm{mg} / \mathrm{dL}$ ); condición de fumador activo (actual o en el último año), fumador antiguo (entre 1-5 años); insuficiencia renal (creatinina basal $>1,5 \mathrm{mg} / \mathrm{dL}$ ); procedimientos de revascularización coronaria previa; antecedente de infarto antiguo ( $>30$ días), infarto reciente (entre 1 y 30 días) o agudo (IAM, en las primeras $24 \mathrm{~h}$ ); forma de presentación del cuadro anginoso como 
estable, inestable o isquemia silente; número de vasos coronarios enfermos (estenosis $>50 \%$ ), función ventricular izquierda estimada o medida en ventriculografía de contraste, radioisotópica o ecocardiografía como normal $=$ fracción de eyección $>50 \%$, leve-moderadamente disminuida $=$ fracción de eyección 30-50\%, acentuadamente deprimida $=$ fracción de eyección $<30 \%$.

La información del procedimiento recopilada fue: número de vasos y lesiones o segmentos coronarios tratados; tamaño de vaina o introductor arterial empleado; dosis de heparina; uso de aspirina, thienopiridínicos, antagonistas de la glicoproteína IIb IIIa de las plaquetas, inótropos o vasopresores y uso de balón de contrapulsación intraaórtica.

Al momento del alta hospitalaria se registró el pico máximo alcanzado por la fracción $\mathrm{MB}$ de la creatinfosfokinasa (CPK-MB); ocurrencia fuera del laboratorio de oclusión aguda del vaso tratado; ocurrencia de nuevo infarto agudo del miocardio con o sin supradesnivel del segmento ST (elevación de CPK-MB mayor a 3 veces valor máximo normal); realización de nueva $\mathrm{AC}$ en otro o el mismo vaso tratado; realización de cirugía de puente coronario electiva o urgente (en las primeras $24 \mathrm{~h})$.

La información angiográfica basal y post angioplastia, así como el tratamiento efectuado, fue recopilado en forma separada para cada una de las lesiones coronarias que se trataron o se intentó tratar durante el procedimiento. Se registró en cada centro: vaso tratado; localización y tratamiento previo de la lesión (angioplastia con o sin stent); clasificación de la lesión de acuerdo al esquema del American College of Cardiology/ American Heart Association (ACC/AHA) en lesiones tipo A, B1, B2 y C ${ }^{9}$; presencia en la angiografía basal de trombo angiográficamente visible, lesión de bifurcación (con rama adyacente de $>2$ $\mathrm{mm}$ y lesión ostial $>50 \%$ ) y oclusión total (corte abrupto del vaso con flujo anterógrado TIMI (Thrombolysis in Myocardial Infarction)) 0-1; flujo TIMI basal y final ${ }^{10}$; estimación visual del grado de estenosis en forma basal y al finalizar el procedimiento. Se registró también el tipo de procedimiento efectuado y el diámetro máximo de balón o stent empleado. Se definió como éxito angiográfico de la lesión una estenosis final $<50 \%$ y flujo TIMI final 2-3. En caso contrario, se determinó el fracaso angiográfico. Se registró la causa específica del fracaso angiográfico y la ocurrencia de complicaciones angiográficas no necesariamente causantes del fracaso. Los resultados se expresan como frecuencias y porcentaje en el caso de las variables discontinuas y como promedio \pm desviación estándar en el caso de las continuas.

Se definió como éxito clínico la obtención de éxito angiográfico en al menos una lesión tratada (éxito angiográfico completo o parcial) en ausencia de complicaciones isquémicas mayores: muerte de cualquier causa, infarto con supradesnivel del ST, oclusión aguda del vaso tratado y nueva revascularización al vaso tratado con angioplastia o cirugía (siempre y cuando hubiese estenosis $>50 \%$ en ese vaso).

\section{RESULTADOS}

Se ingresaron al registro un total de 1.484 procedimientos de AC, 1.143 hombres y 341 (22,98\%) mujeres. La edad de los pacientes fue de 60,8 \pm 11,3 años con un rango de 23 a 94 años. En la Tabla 1 se muestran las características clínicas basales de la totalidad de los pacientes y el subgrupo sin IAM donde se excluyen los pacientes sometidos a angioplastia en las primeras $24 \mathrm{~h}$ de evolución de un infarto agudo al miocardio. Entre los factores de riesgo destaca una frecuencia de $21,8 \%$ de diabetes mellitus y una alta incidencia de hipertensión arterial (60\%), dislipidemia (52\%) y tabaquismo activo (40\%). La minoría de los pacientes tenía procedimientos de revascularización coronaria previos, 9,1\% cirugía y 16,4\% angioplastia. La mayoría de los pacientes tenía un síndrome coronario agudo, $46 \%$ angina inestable, $19,7 \%$ infarto agudo y $28,4 \%$ infarto reciente al miocardio. Los pacientes con angina estable $(12,1 \%)$ o isquemia miocárdica silente $(3,2 \%)$ fueron infrecuentes.

La mayoría correspondió a enfermedad de un vaso $(53,1 \%)$ y alrededor de un tercio a enfermedad de 2 vasos (30,8\%). La gran mayoría de los pacientes tenía una función sistólica global del ventrículo izquierdo normal (70,8\%), siendo muy 
Tabla 1. Características clínicas basales

\begin{tabular}{|c|c|c|}
\hline Variable & $\begin{array}{c}\text { Todos } \\
(\mathrm{n}=1.484)^{\&}\end{array}$ & $\underset{(n=1.192)^{\&}}{\operatorname{Sin} \text { IAM }}$ \\
\hline Sexo masculino & $1.143(77,0)$ & $914(76,7)$ \\
\hline Edad (años) & $60,7 \pm 11,3$ & $60,9 \pm 11,1$ \\
\hline Diabetes Mellitus & $323(21,8)$ & $259(21,7)$ \\
\hline Hipertensión arterial & $894(60,2)$ & $754(63,3)$ \\
\hline Dislipidemia & $772(52,0)$ & $647(54,3)$ \\
\hline Tabaquismo activo & $593(40,0)$ & $453(38)$ \\
\hline Tabaquismo antiguo & $106(7,1)$ & $88(7,4)$ \\
\hline Insuficiencia renal & $92(6,2)$ & $80(6,7)$ \\
\hline Cirugía coronaria previa & $135(9,1)$ & $122(10,2)$ \\
\hline Angioplastia coronaria previa & $244(16,4)$ & $221(18,5)$ \\
\hline Infarto antiguo al miocardio & $324(21,8)$ & $295(24,8)$ \\
\hline Infarto reciente al miocardio & $421(28,4)$ & $417(35,0)$ \\
\hline Infarto agudo al miocardio & $292(19,7)$ & - \\
\hline Angina estable & $180(12,1)$ & $180(15,1)$ \\
\hline Angina inestable & $682(46,0)$ & $682(57,2)$ \\
\hline Isquemia miocárdica silente & $47(3,2)$ & $47(3,9)$ \\
\hline \multirow[t]{3}{*}{$\mathrm{N}^{\circ}$ vasos enfermos: } & $783 / 1.475(53,1)$ & $644 / 1.186(54,3)$ \\
\hline & $454 / 1.475(30,8)$ & $364 / 1.186(30,7)$ \\
\hline & $238 / 1.475(16,1)$ & $178 / 1.186(15,0)$ \\
\hline \multicolumn{3}{|l|}{ Función ventricular izquierda: } \\
\hline Normal & $979 / 1.383(70,8)$ & $831 / 1.109(74,9)$ \\
\hline Depresión leve/moderada & $353 / 1.383(25,5)$ & $248 / 1.109(22,4)$ \\
\hline Depresión acentuada & $51 / 1.383(3,7)$ & $30 / 1.109(2,7)$ \\
\hline
\end{tabular}

\&En algunos casos los totales pueden no sumar el total de casos, debido a información no entregada. Los porcentajes entre paréntesis se calcularon sobre el número de casos informados. «n el caso de la edad, los valores se expresan como promedio \pm desviación estándar».

infrecuente la disfunción sistólica global acentuada $(3,7 \%)$.

Se trataron un total de 1.628 vasos (1,1 vaso/ procedimiento) y 1.768 lesiones coronarias $(1,2$ lesiones/procedimiento). Se utilizó balón solamente en 317 (18,0\%) lesiones, stent en 1.442 $(81,7 \%)$ lesiones, de los cuales $447(25,3 \%)$ se implantaron sin predilatación, 994 luego de dilatación con balón y 1 caso luego de aterectomía rotacional. La aterectomía rotacional se empleó sólo en 6 y la direccional en 1 lesión. Todos los procedimientos de aterectomía correspondieron a procedimientos en pacientes sin IAM. Como farmacoterapia coayudante, la gran mayoría recibió aspirina o thienopiridínicos. Casi 20\% recibió algún antagonista IIb IIIa, proporción que desciende a $13,2 \%$ en los procedimientos sin IAM, como se aprecia en la Tabla 2. En la Tabla 3 se 
Tabla 2. Características del procedimiento

\begin{tabular}{|llcc|}
\hline Variable & & $\begin{array}{c}\text { Todos } \\
(\mathrm{n}=1.484)^{\&}\end{array}$ & $\begin{array}{c}\text { Sin IAM } \\
(\mathrm{n}=1.192)^{\&}\end{array}$ \\
\hline \multirow{2}{*}{$\mathrm{N}^{\circ}$ vasos tratados: } & 1 & $1.344(90,6)$ & $1.067(89,6)$ \\
& 2 & $133(9,0)$ & $118(9,9)$ \\
& 3 & $6(0,4)$ & $6(0,5)$ \\
$\mathrm{N}^{\circ}$ lesiones tratadas: & 1 & $1.209(81,5)$ & $961(80,7)$ \\
& 2 & $240(16,2)$ & $202(16,9)$ \\
& 3 & $32(2,2)$ & $26(2,2)$ \\
Tamaño introductor: & 6 French & $2(0,1)$ & $2(0,2)$ \\
& 7 French & $1.178 / 1.474(80,1)$ & $930 / 1.182(78,8)$ \\
& $\geq 8$ French & $237 / 1.474(16,1)$ & $201 / 1.182(17,0)$ \\
Heparina sódica UI & & $56 / 1.474(3,8)$ & $50 / 1.182(4,2)$ \\
Aspirina & & $8.255 \pm 2.289$ & $8.235 \pm 3.099$ \\
Ticlopidina & & $1.449(97,6)$ & $1.168(98,0)$ \\
Clopidogrel & & $71(4,8)$ & $65(5,5)$ \\
Tirofiban & & $1.303(87,9)$ & $1.061(89,1)$ \\
Abciximab & $145(9,8)$ & $108(9,1)$ \\
Epifibatide & & $140(9,4)$ & $45(3,8)$ \\
Vasopresores/inótropos & $5(0,3)$ & $3(0,3)$ \\
Balón de contrapulsación intraórtico & $78(5,3)$ & $26(2,2)$ \\
& & $33(2,2)$ & $9(0,8)$ \\
\hline
\end{tabular}

\& En algunos casos los totales pueden no sumar el total de casos, debido a información no entregada. Los porcentajes entre paréntesis se calcularon sobre el número de casos informados.

Tabla 3. Resultados clínicos intrahospitalarios

\begin{tabular}{|llcc|}
\hline Variable & & $\begin{array}{c}\text { Todos } \\
(\mathrm{n}=1.484)\end{array}$ & $\begin{array}{c}\text { Sin IAM } \\
(\mathrm{n}=1.192) \&\end{array}$ \\
\hline Razón CPK-MB máxima: & $0-1 \mathrm{x}$ & & $661 / 898(73,6)$ \\
& $1-3 \mathrm{x}$ & & $185 / 898(20,6)$ \\
& $3-5 \mathrm{x}$ & & $25 / 898(2,8)$ \\
& $>5 \mathrm{x}$ & & $27 / 898(3,0)$ \\
Nueva oclusión aguda & & $15(1,0)$ & $11(0,9)$ \\
Nuevo IAM con SDST & & $6(0,4)$ & $5(0,4)$ \\
Nuevo IAM sin SDST & & $55(3,7)$ & $52(4,4)$ \\
Nueva angioplastia: & Al mismo vaso & $21(1,4)$ & $15(1,3)$ \\
& A otro vaso & $21(1,4)$ & $8(0,7)$ \\
Nueva cirugía coronaria: & Electiva & $11(0,7)$ & $6(0,5)$ \\
& Urgente & $7(0,5)$ & $4(0,3)$ \\
Muerte & & $32(2,2)$ & $13(1,1)$ \\
Éxito clínico & & $1.379(92,2)$ & $1.133(95,1)$ \\
\hline
\end{tabular}

\& En algunos casos los totales pueden no sumar el total de casos, debido a información no entregada. Los porcentajes entre paréntesis se calcularon sobre el número de casos informados. 
aprecian las características angiográficas basales y post procedimientos. Los vasos más frecuentemente tratados fueron la arteria descendente anterior $(42,1 \%)$, la arteria coronaria derecha $(31,5 \%)$ y la arteria circunfleja (23\%), siendo infrecuente las lesiones en el tronco de la coronaria izquierda y en los puentes venosos o arteriales. Una minoría de las lesiones había sido tratada previamente, 3,8\% con balón y 3,3\% con stent. De acuerdo a la clasificación del ACC/AHA 57,9\% correspondía a lesiones complejas $\left(\mathrm{B}_{2}\right.$ y $\left.\mathrm{C}\right)$ y $24,9 \%$ presentaban oclusión total. El grado de estenosis se redujo de 85,3 $\pm 13,9$ a $8,3 \pm 19,5 \%$ luego del procedimiento. Similarmente el flujo TIMI se mantuvo o incrementó en la mayoría de las lesiones. El 90,4\% finalizó con flujo TIMI grado 3 , cifra que fue $93,4 \%$ en el grupo sin IAM. Se obtuvo éxito angiográfico en 95,3\% de las lesiones tratadas y en $96,2 \%$ en las tratadas electivamente. Las causas de fracaso angiográfico fueron por orden de frecuencia: en $30(1,7 \%)$ casos la guía no cruzó la lesión, $14(0,79 \%)$ no reflujo o macroembolización masiva, $12(0,68 \%)$ lesión no dilatable, $12(0,68 \%)$ retracción elástica acentuada con estenosis residual mayor a 50\%, $11(0,62 \%)$ disección residual con estenosis residual mayor a $50 \%$ y en 4 $(0,23 \%)$ lesiones no se logró intubar la coronaria con el catéter guía.

En la Tabla 4 se muestran los resultados clínicos intrahospitalarios. La mortalidad global fue $2,2 \%$, pero sólo $1,1 \%$ en pacientes que no cursaban un infarto agudo. Complicaciones como la oclusión aguda, infarto agudo con SDST, necesidad de cirugía o nueva AC fueron infrecuentes. El éxito clínico global fue 92,2\%, pero $95,1 \%$ en los casos sin IAM. Entre los procedimientos sin IAM hubo infarto del miocardio con supradesnivel del ST en sólo 5 casos $(0,4 \%)$, siendo más frecuente el infarto sin supradesnivel del ST en 52 $(4,4 \%)$ casos. Alzas menores de la CPK-MB, entre 1 y 3 veces el valor máximo normal, fueron frecuentes, $185(20,6 \%)$ entre los 898 pacientes que informaron las enzimas.

\section{Discusión}

La población estudiada no corresponde al total de angioplastias coronarias realizadas en el país en el período de un año, como fue nuestra intención original, sin embargo, es muy representativa de la misma, ya que de acuerdo a estimaciones, representa a más de $50 \%$ de los pacientes tratados en el período de estudio y en segundo lugar es una muestra que refleja la práctica en diferentes tipos de instituciones de salud: hospitales universitarios, pertenecientes al Sistema de Servicios de Salud, de las Fuerzas Armadas y clínicas privadas.

La edad promedio es muy similar a otros estudios nacionales y extranjeros. Sin embargo, la proporción de mujeres es menor a 32-34\% publicada por los registros norteamericanos, pero similar a registros europeos ${ }^{11-13}$. La prevalencia de factores de riesgo es alta, destacando la HTA y la dislipidemia que afectan a más de $50 \%$ de nuestros pacientes. La diabetes alcanza a casi $22 \%$ de la población, algo menor que $26 \%$ observado en Estados Unidos de América (EEUU), cifra que iría en aumento ${ }^{11,12}$.

Existe una baja frecuencia de procedimientos de revascularización previos como AC y cirugía de revascularización miocárdica, siendo aproximadamente en las 3/4 partes el procedimiento estudiado el primer procedimiento de revascularización. Estas cifras contrastan con el registro del ACC-NCDR, en que el antecedente de revascularización previa percutánea o quirúrgica es el doble más frecuente que en este estudio ${ }^{11}$. La gran mayoría de los pacientes son tratados por síndromes coronarios agudos. Los procedimientos en pacientes anginosos estables o con isquemia silente son la excepción, a diferencia de los registros norteamericanos donde superan $25 \%$ de los pacientes ${ }^{11,12}$.

La mayoría de los pacientes tiene función sistólica del ventrículo izquierdo normal, la cuarta parte de los pacientes tiene una disfunción sistólica leve o moderada, siendo excepcional la disfunción sistólica acentuada (fracción de eyección $<30 \%$ ), al igual que las series norteamericanas. La mayoría tiene enfermedad de un vaso, casi un tercio enfermedad de dos vasos y la enfermedad de tres vasos es poco frecuente, a diferencia de los registros norteamericanos que incorporan una mayor proporción de pacientes con enfermedad de 3 vasos (21-23\%) y una menor con enfermedad de 1 vaso $(41-46 \%)^{11,12}$. Al igual que en las series norteamericanas, en nuestro estudio lo más frecuente es el tratamiento de solo un vaso $(86,5 \%$ y 
Tabla 4. Información angiográfica pre y post angioplastia

\begin{tabular}{|c|c|c|}
\hline Variable & $\begin{array}{c}\text { Todos } \\
(\mathrm{n}=1.768)\end{array}$ & $\begin{array}{l}\text { Sin IAM agudo } \\
\quad(\mathrm{n}=1.424)\end{array}$ \\
\hline Vaso tratado: Tronco coronaria izquierda & $17(0,9)$ & $15(1,0)$ \\
\hline Descendente anterior & $744(42,1)$ & $587(41,2)$ \\
\hline Circunfleja & $406(23,0)$ & $344(24,2)$ \\
\hline Coronaria derecha & $557(31,5)$ & $440(30,9)$ \\
\hline Puente venoso & $37(2,1)$ & $32(2,3)$ \\
\hline Puente arterial & $7(0,4)$ & $6(0,4)$ \\
\hline Localización lesión: Ostial & $64(3,6)$ & $51(3,6)$ \\
\hline Proximal & $610(34,5)$ & $479(33,7)$ \\
\hline Medio-distal & $1.071(60,7)$ & $875(61,5)$ \\
\hline Anastomosis & $21(1,2)$ & $17(1,2)$ \\
\hline Tratamiento previo lesión tratada: No & $1.643(92,9)$ & $1.315(92,4)$ \\
\hline Balón & $67(3,8)$ & $62(4,4)$ \\
\hline Stent & $58(3,3)$ & $47(3,3)$ \\
\hline Clasificación ACC/AHA: A & $168(9,5)$ & $161(11,3)$ \\
\hline B1 & $574(32,5)$ & $446(31,4)$ \\
\hline B2 & $765(43,2)$ & $608(42,8)$ \\
\hline $\mathrm{C}$ & $259(14,7)$ & $207(14,6)$ \\
\hline Trombo angiográfico & $317(17,9)$ & $172(12,1)$ \\
\hline Lesión bifurcación & $221(12,5)$ & $178(12,5)$ \\
\hline Oclusión total & $441(24,9)$ & $201(14,1)$ \\
\hline Tamaño máximo balón/stent (mm) & $3,1 \pm 0,6$ & $3,1 \pm 0,6$ \\
\hline Flujo TIMI basal/final: 0 & $388(22,0) / 42(2,4)$ & $163(11,5) / 27(1,9)$ \\
\hline 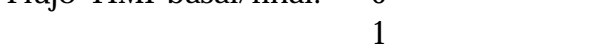 & $94(5,3) / 14(0,8)$ & $71(5,0) / 7(0,5)$ \\
\hline 2 & $291(16,5) / 114(6,5)$ & $243(17,1) / 60(4,2)$ \\
\hline 3 & $994(56,3) / 1.596(90,4)$ & $946(66,5) / 1.328(93,4)$ \\
\hline Grado estenosis \% basal & $85,3 \pm 13,9$ & $83,3 \pm 13,7$ \\
\hline Grado estenosis \% final & $8,3 \pm 19,5$ & $7,5 \pm 18,1$ \\
\hline Éxito angiográfico & $1.684(95,3)$ & $1.370(96,2)$ \\
\hline
\end{tabular}

$90,6 \%$, respectivamente). Sin embargo, en esas series se tratan con mayor frecuencia 2 ó 3 vasos durante la $\mathrm{AC}$, siendo este caso la excepción en Chile. De acuerdo a estos antecedentes, hay vasos enfermos que no fueron revascularizados. No conocemos las razones por las cuales no lo fueron, pero una pequeña proporción de pacientes fueron tratados por otros vasos en un segundo procedimiento de revascularización durante la hospitalización. Es posible que otros vasos no fuesen revascularizables o tuviesen lesiones con grados menores de estenosis significativas (50\%$70 \%$ ) que no contribuyesen al cuadro clínico.

La mayoría de los pacientes son pretratados con aspirina, pero en esta serie sólo una proporción levemente menor con thienoperidinas (93\%), esperable en una población con un muy alto uso de stents ( $81 \%$ de las lesiones tratadas). Entre ellas, 
el clopidogrel es la más usada, probablemente por el mejor perfil de seguridad con relación a ticlopidina $^{14}$. En cambio, el uso de los antagonistas IIb IIIa es bajo, considerando la alta prevalencia de los síndromes coronarios agudos, donde estas drogas han mostrado sus mayores beneficios. Es probable que su alto costo haya incidido en el bajo uso.

Como es habitual, el vaso tratado con mayor frecuencia fue la arteria descendente anterior, seguido de la arteria coronaria derecha y la circunfleja, siendo la mayoría de las lesiones de novo. El tratamiento de lesiones del tronco de la coronaria izquierda, como de puentes coronarios, fue poco frecuente. Esto es similar a lo observado en EEUU, siendo algo más frecuente allá el tratamiento de puentes y de la arteria descendente anterior en menor proporción ${ }^{11,12}$.

De acuerdo a la calificación de los investigadores la mayoría de las lesiones fueron clasificadas como complejas (B2+C en 58\%), habiendo con frecuencia características específicas de complejidad como oclusión total, trombo y bifurcaciones. A pesar de ello se alcanzó el éxito angiográfico en la gran mayoría, $95,3 \%$ en toda la población y $96,2 \%$ si se excluyen los infartos agudos. Esto se compara favorablemente con el 93,7\% obtenido en el registro del NHLBI 1997-1998 y el 94,5\% obtenido en el registro del ACC-NCDR ${ }^{11,12}$.

El éxito clínico fue obtenido en un alto porcentaje, en particular en los casos sin IAM. Es algo menor en el grupo total (92,2\%), puesto que se incluyen los pacientes con infarto agudo. Esto es muy similar al $92 \%$ obtenido en los registros norteamericanos ${ }^{11,12}$. Sin embargo, nuestra definición de éxito clínico es más exigente al considerar la oclusión aguda y la revascularización no urgente de la lesión como causa de fracaso clínico.

La mortalidad global de 2,2\% está importantemente influida por la mortalidad de los pacientes con infarto agudo del miocardio. De hecho, de los 32 fallecidos, 19 lo hicieron en el contexto de angioplastia en las primeras $24 \mathrm{~h}$ de un infarto del miocardio. Sólo 13, fallecieron en procedimientos sin IAM. Sin embargo, no podemos descartar, que algunas de estas muertes hayan ocurrido en el contexto de angina inestable refractaria o infarto reciente del miocardio con 0 sin inestabilidad hemodinámica. Esta tasa de mortalidad es superior a $1,4 \%$ del registro del ACC-NCDR y al 0,5\%-0,6\% obtenido en Europa en general y en Dinamarca en particular. Sin embargo, es similar a 1,9\% del registro del NHLBI. Hay que considerar que estos registros tienen una menor proporción (alrededor de 10\%) de infarto agudo del miocardio. Con ese considerando, nuestra cifra de mortalidad es muy similar a los registros norteamericanos no así a la de los registros europeos ${ }^{11-13,15}$.

En cuanto a otros componentes individuales de éxito clínico, la oclusión aguda fue menos frecuente en nuestra serie (1,0 vs 1,9\%) y el infarto del miocardio con supradesnivel del ST fue igualmente infrecuente con relación al registro del ACC-NCDR ${ }^{11}$.

Entre los pacientes en que se registraron las enzimas con frecuencia hubo alza de CPK-MB. En 52, hubo alzas sobre 3 veces el valor normal y fueron catalogadas de infarto agudo del miocardio. En 185, se registraron alzas menores de CPK$\mathrm{MB}$, entre 1 y 3 veces el valor máximo normal. Esto corresponde a $20,6 \%$ de la población estudiada, cifra elevada con relación a otras experiencias. Sin embargo, a casi 300 pacientes no se les registraron enzimas. Es probable que haya habido algún sesgo en la toma de enzimas a los pacientes más graves o con complicaciones.

La realización de nuevos procedimientos de revascularización fue infrecuente, en particular la cirugía. Especialmente infrecuente fue la cirugía de urgencia, que se practicó en aproximadamente 1 de cada 300 pacientes sin IAM. Estas cifras son similares, pero levemente menores a los registros norteamericanos ${ }^{11,12}$, pero levemente mayores al 0,3\% reportado en Europa ${ }^{15}$.

En conclusión, en Chile la angioplastia coronaria percutánea se efectúa principalmente para el tratamiento de los síndromes coronarios agudos. El stent es el dispositivo usado en la gran mayoría de los casos. Los resultados intrahospitalarios obtenidos son comparables en muchos aspectos a lo publicado en los registros estadounidenses, sin embargo, la mortalidad es mayor que lo publicado en Europa. No queda claro sin embargo, si estas diferencias son atribuibles a diferencias en las poblaciones tratadas. 


\section{REFERENCIAS}

1. Pichard A, Marchant E, Casanegra P, Rodríguez J, Chamorro G, Corbalán R et al Angioplastia transluminal percutánea en un paciente con arterioesclerosis coronaria. Rev Méd Chile 1983; 111: 709-13.

2. Dussailant $G$, Martínez A, Marchant E, Fajuri $A$, CASTRO P, CORBalÁN R. Angioplastia comonaria primaria como tratamiento de reperfusión precoz del infarto agudo del miocardio. Rev Méd Chile 1994; 122: 401-7.

3. Chamorro $\mathrm{H}$, Ducci $\mathrm{H}$, Matthei R, Alcaíno $\mathrm{M}$, Florenzano F, Ramírez A et al. Angioplastia coronaria primaria como tratamiento de elección en las primeras 6 horas del infarto de miocardio. Rev Méd Chile 1995; 123: 727-34.

4. Marchant E, Martínez A, Fajuri A, Guarda E. Angina inestable: resultados inmediatos y a un año del tratamiento con angioplastia coronaria transluminal percutánea. Rev Méd Chile 1998; 126: 1338-44.

5. Ugaide $\mathrm{H}$, Ramírez A, Benavente $\mathrm{D}$, Antúnez $\mathrm{M}$, García S, Dussailant G et al. Angioplastia en infarto agudo de miocardio: resultado inicial y seguimiento alejado. Rev Méd Chile 1999; 127: 565-75.

6. Dussailant $G$, Pacheco C, Ramírez A, Ugalde $H$, Antibilo S, Silva AM et al. Estudio comparativo de la angioplastia coronaria en pacientes con diabetes. Resultados clínicos y angiográficos inmediatos y evolución clínica en el primer año. Rev Méd Chile 2001; 129: 861-70.

7. Dussailant $G$, Ayala F, Ramírez A, Ugalde $H$, Silva A, Farías E et aL. Implante de prótesis endovasculares (stents) en el tratamiento de la cardiopatía coronaria: resultados angiográficos, clínicos inmediatos y tardíos. Rev Méd Chile 1998; 126: 1195-205.
8. Marchant E, Martínez A, Fajuri A, Guarda E, ValenZuela E, Cortés N et al. Aterectomía rotacional (Rotablator) en la angioplastia coronaria percutánea transluminal. Rev Méd Chile 1997; 125: 1474-82.

9. Euns SG, Vandormael MG, Cowley MJ, Disciascio G, Deligonul U, Topol EJ et al. Coronary Morphological and Clinical Determinants of Procedural Outcome with Angioplasty for Multivessel Coronary Disease: Implications for Patient Selection. Circulation 1990; 82: 1193-202.

10. The TIMI Study Group. The thrombolysis in myocardial infarction (TIMI) trial. N Engl J Med 1985; 31: 932-6.

11. Anderson HV, Shaw RE, Brindis RG, Hewitt K, KRONE RJ, BLoCK PC ET AL. A contemprary overview of percutaneous coronary interventions. The American College of Cardiology-National Cardiovascular Data Registry (ACC-NCDR). J Am Coll Cardiol 2002; 39: 1098-103.

12. Wiwams DO, Holubkov R, Yeh W, Bourassa MG, Al-Bassam M, Block PC et al. Percutaneous Coronary Intervention in the Current Era Compared With 1985-1986. Circulation 2000; 102: 2945-51.

13. Jensen LO, Thayssen P, Kassis E, Rasmussen K, Saunamaki K, Thuesen L. Percutaneous coronary intervention in Denmark from 1989 to 1998. Results from the Danish PTCA registry. Ugeskr Laeger 2003; 165: 2809-12.

14. Bertrand ME, Rupprecht HJ, Urban P, Gershlick AH FOR THE CLASSICS InVESTIGATORs. Double-Blind Study of the Safety of Clopidogrel With and Without a Loading Dose in Combination With Aspirin After Coronary Stenting. The Clopidogrel Aspirin Stent International Cooperative Study (CLASSICS). Circulation 2000; 102: 624-9.

15. Rotter M, Pfiffner D, Maier W, Zeiher AM, Meier B. Interventional cardiology in Europe 1999. Eur Heart J 2003; 24: 1164-70.

APÉNDICE 1

Hospital de la Fuerza Aérea de Chile, Santiago, Dr. Juan Delgado (investigador), M Galilea (coordinador); Hospital Clínico Universidad Católica, Santiago, Dr. Eugenio Marchant y Dr. Alejandro Fajuri (investigadores), A Alcayaga (coordinador); Hospital San Juan de Dios, Santiago, Dr. Hernán Donoso (investigador), M Urqueta (coordinador); Hospital Clínico 
Universidad de Chile, Santiago, Dr. Gastón Dussaillant (investigador); Hospital Gustavo Fricke, Valparaíso, Dr. Mauricio Aninat (investigador); Hospital Naval, Viña del Mar, Dr. Marcos Opazo (investigador), P Lavín (coordinador); Clínica Las Condes, Santiago, Dr. Ronald Kauffmann (investigador), B Muñoz (coordinador); Hospital Militar, Santiago, Dr. René Pumarino (investigador), N Espinoza (coordinador); Hospital Fundación de Salud El Teniente, Rancagua, Dr. Gastón Dussaillant (investigador), F Huerta (coordinador); Hospital Regional de Temuco y Clínica Alemana de Temuco, Dr. Jorge Escobar (investigador). 